\title{
Plant Disease Detection through the Implementation of Diversified and Modified Neural Network Algorithms
}

\author{
Fatema Nihar, Nazmun Nahar Khanom, Syed Sahariar Hassan, Amit Kumar Das* \\ Department of Computer Science and Engineering, East West University, Dhaka, Bangladesh
}

Received: January 28, 2021, Revised: February 25, 2021, Accepted: March 01, 2021, Available Online: March 12, 2021

\begin{abstract}
In the era of artificial systems, disease detection is becoming easier. For detecting disease, monitoring the plants 24 hours, visiting the agricultural office, or asking for help from a specialist seem difficult. This situation demands a user-friendly plant disease detection system, which allows people to detect whether the plant is diseased or not in an easier way. If the plant is diseased, a treatment plan will also be notified. In this way, people can easily save time, money, and, most importantly, plants. In this study, the researchers have collected data of vegetables from a field and applied multiple diversified Neural Network Algorithms such as CNN, MCNN, FRCNN, and, along with that, also proposed a new modified neural network architecture (ModCNN), which has produced 97.69\% accuracy. The authors have also classified the bean leaf diseases into four categories according to their symptoms, which will help to identify diseases accurately.
\end{abstract}

Keywords: Artificial, Agriculture, Monitoring, Disease Detection, Neural Network.

This work is licensed under a Creative Commons Attribution-Non Commercial 4.0 International License.

\section{Introduction}

As days go by, people face different types of problems regarding plant disease. Some may know the reason and treatment, but most people do not. People tend to browse online to find out the answers. However, what if the answer can be found just by uploading a photo? In this way, people will not only know the cause, types of the disease but also will be able to know about the treatment. This process will be time, cost-effective and allow people to interact more and gain valuable information about plants.

At present, it is comprehensible to design such a system that can interact with people more effectively. Different types of organizations such as Bangladesh Rice Research Institute, SherE-Bangla Agricultural University, etc., are researching continuously to discover new findings for different kinds of crops. The objective of this research is to detect plant diseases. Moreover, this research study has classified the bean leaf disease into four categories: low, mid, high, and fully infected. It will specify how much the disease has spread through the leave, and it will notify the user to take fruitful steps. These will help the agriculture field advance towards development, and the researchers will contribute more. Several studies related to this topic have been done before. In paper [1], the authors have used 50,000 images to classify plant disease.

In contrast, in paper [2], the authors have combined three color components in a three-layer convolutional network to address plant disease accurately. In another paper [3], the authors have also worked with 14 different crops and deliver the highest accuracy in finding the disease. Therefore, it has been seen that this research study provides slightly less accuracy than some of the other research studies. Nevertheless, in this study, the researchers differentiated the diseases and identified the disease more specifically. The researchers have built their own CNN architecture that gives higher accuracy among the algorithms the authors have used so far.
In paper [4], the authors have used a cloud-based platform to track the disease easily for farmers. In paper [5], the authors have detected early leaf disease to ensure efficient crop production. In paper [6], the authors have worked with 14 different crops with distinct 25 diseases, which gives high accuracy results.

There are other related works as well, which intend to perform disease detection and provide good results.

\section{Background}

In paper [1], the authors have classified and recognized plant disease. At most, 50,000 images have been used in the database. CNN, with GoogLeNet architecture, has been implemented here with augmented training datasets. Plant pathologists' and farmers' participation will improve the process for collecting the images, image labeling, and other parts of the plants such as root, stems, and so on that will produce different future results.

In paper [2], the authors have depicted vegetable leaf disease's recognition process combining three-color components using a three-channel convolutional network (TCCNN) model.

In paper [3], the authors have used deep learning models to identify plant disease. To train the architectures, Keras with Theano backend have been used. In this paper, the authors have used VGG16, Inception v4, ResNet with 50,101,152 layers, and DenseNets with 121 layers for the model.

In paper [4], the authors have implemented the $\mathrm{CNN}$ model and a cloud-based platform to identify and track farmers' plant disease. In the future, using different factors such as soil, pesticide treatment, temperature, rainfall, etc., will improve the accuracy of the model and will help to enable disease forecasting.

In paper [5], the authors have detected early leaf disease for the efficient production of crops. CNN and Learning Vector Quantization (LVQ) algorithms have been implemented with three channels (RGB components) based filters.

In paper [6], the authors have used AlexNet, GoogLeNet architecture with a transfer learning approach for building the model for detecting the disease of plants using leaves images. 
Image collection using realistic methods will produce different results in the future.

In paper [7], the authors have implemented architectures of CNN, such as AlexNet, AlexNetOWTBn, Overfeat, VGG, and GoogLeNet, to build the model for detection and diagnosis of the disease of the plants. Automated pesticide prescription systems will allow the farmers to purchase the appropriate pesticides in the future.

In paper [8], the authors have diagnosed the early detection of apple leaf diseases by implementing GoogLeNet Inception architecture and Rainbow Concatenation, INAR-SSD (Singleshot multi-box detection with Rainbow Concatenation and inception module).

In paper [9], the authors have developed a multilayer convolutional neural network (MCNN) to predict healthy or diseased mango tree leaves. At most, 1070 real-time healthy or diseased Mango tree leaf images and 1130 images from plant village datasets have been used. The authors have detected fungal disease-Anthracnose of Mango tree leaves. Their proposed model has given $97.13 \%$ accuracy, which is higher than the other approaches. In the future, working with other plants with economic importance will produce different results.

In [10], [11], [12], [13] and [14] the authors have applied Faster R-CNN with FCM-KM fusion for the rapid rice blast, bacterial blight, and blight disease detection. The authors have also used the 2DFM-AMMF algorithm for noise reduction and faster 2D-Otsu segmentation. The authors have applied a max and min distance algorithm for the optimization of FCM-KM. The average accuracy found from the proposed model is $97.2 \%$. In the future, automated rice disease detection using mobile will allow real-time monitoring and pest identification.

In the paper [15], the authors have used K-means clustering and support vector machine algorithms to predict and classify soybean leaf disease. The diseased leaves are divided into Septoria leaf blight, downy mildew, and frog eye. Four thousand seven hundred seventy-five images of plant village datasets have been taken. The average classification accuracy found from the model is almost $90 \%$. In the future, using real-time images will bring a difference to the result.

\section{Main Focus of the Article}

Numerous researches have been done to detect plant diseases, but the researchers have applied either existing algorithms or proposed algorithms. The main goal behind this is to increase accuracy as much as possible to produce an accurate result. In paper [1], the author has applied CNN with GoogleNet architecture on a database that contains 50,000 images of 171 diseases affecting 21 plant species. Still, the author has only used corn disease due to its' widest variety of conditions. The author has been able to achieve only $87 \%$ accuracy. Still, their future task of involving farmers and plant pathologists in image collection has been one of the inspirations behind this study. In paper [5], the authors have applied CNN with the Learning Vector Quantization algorithm on 500 images of Tomato with four symptoms of diseases and have achieved only $86 \%$ accuracy. However, while going through the detection phase, the authors have faced issues such as some of the images being labeled in the wrong classes due to their similarities. In this study, the researchers have used CNN, MCNN, FRCNN, and ModCNN to detect the diseases according to classes accurately. Paper [1] and paper [5] have worked as good influences. This study focuses on proposing a new algorithm to produce the highest accuracy to detect plant diseases accurately and involve people from all occupations in this process.

The data, which will be collected from the user, will be processed thoroughly to predict the disease. The data collected from the user can be the image of the plant. This disease prediction will help to reduce costs in the future, minimize treatment costs and unexpected expenses.

Using the prediction outcome, people do not need to wait for any agricultural officers or expert opinions. Instead, they can immediately start taking preventive measures and treatment actions if required. Nevertheless, for advanced cases of plants, experts' advice is highly recommendable. Therefore, this study's proposed prediction model helps people save plants and provide better services. It also reduces the time of diagnosis and unnecessary cost of treatment.

\section{Methods and Materials}

\subsection{Image processing in plant disease detection}

Through the image processing method, many different types of photos of plants (data) can be diagnosed. These data can be structured or not. However, using this method to detect plant disease and provide information is excellent research to help the agricultural arena. Many people live their livelihood through farming without knowing what type of disease the plant has, what kind of pesticide or treatment to cure the plant. Though there are many agricultural departments and officers to train the farmers, not everybody visits those places to train himself or herself. During bad weather or pandemic, people stay at home to be safe. In this type of situation, people can only use their electronic devices to detect plant disease as nowadays everyone uses a cellphone. In this way, life gets more comfortable, and people can be more productive.

By performing Image processing on an image, one can easily extract the required information about the image. It is one kind of signal processing method, where the image will be the input, and the output can be an image or some characteristics related to that image. As nowadays, the uses of image processing are rapidly increasing, it is also becoming more efficient and valuable to researchers. It is one of the core areas of the research involving three steps.

- Step 1: Using acquisition tools, import the image

- Step 2: Manipulate and analyze the image

- Step 3: Show output where the result is either an altered image or report found in image analysis

In image processing, two kinds of methods are generally used, and they are Analogue and Digital. Analog image processing is mainly used for hard copies such as photographs and printouts, while the digital image processing technique manipulates digital images through computers. All kinds of data undergo three general phases while implementing digital techniques.

- $\quad$ Phase One: The image will be pre-processed

- Phase Two: After enhancing, the image will be displayed

- Phase Three: Extract the required information about the image

Digital Image Processing mainly involves three parts.

- Collection of images.

- Processing of image.

- Analysis of image. 
At the basic level, there are three components in a digital image processing system.

To process images, a computer system.

- An Image Digitizer.

- A Display Device to display image.

There are several essential steps of image processing required to process an image and predict accurate results.

\section{- Step 1: Image Acquisition.}

To produce a digital image, one or more image sensor is needed. Apart from that, different kinds of light-sensitive cameras including, radar, range sensors, ultrasonic cameras, tomography devices, etc., are also required.

\section{- $\quad$ Step 2: Image Enhancement.}

It is mainly used to improve the image's visual quality to bring out the details hidden inside the image. There are also two types of techniques: The transform domain method and the spatial domain method.

The spatial domain Method performs directly on the pixels where the transform domain method only plays on the Fourier transform of an image and transforms it back to the spatial domain.

\section{- $\quad$ Step 3: Image Restoration.}

It is used to remove the noise from the original version of the image. Noise can occur due to camera shaking, less light, etc. In this case, filters are used to remove the noise. Here, after taking a corrupted or noisy image, one can easily estimate the clean original image. Corruption can occur in many different forms like motion blurriness, noises, and missed camera focus.

- $\quad$ Step 4: Morphological Processing.

It can extract components of images that are useful to represent and describe the shape. It is also used for edge detection. It is like a convolution process.

- $\quad$ Step 5: Segmentation.

It partitions the image into a set of pixels. It involves two techniques: Local Segmentation, Global Segmentation.

In Local Segmentation, sub-images are mainly segmented. These images are like small windows in a whole image. The number of pixels that are available to local segmentation is always less than global segmentation.

In Global Segmentation, segmentation of a whole image is performed. The goal of this is to simplify or change the representation part of an image into something else, which can be easily analyzed and will be meaningful too.

\section{- Step 6: Object Recognition.}

It recognizes different parts of an image, such as color, shape, and texture. It can comprehend an object's physical properties and apply semantic attributes to that particular object, which also involves the realization of usage, prior experience with that specific object, and how it relates to the others.

- $\quad$ Step 7: Representation.

One can represent an object by its boundary (its external characteristics) or internal characteristics (texture).

- $\quad$ Step 8: Description.
The object boundary may be described by its length, orientation, or number of concavities.

- $\quad$ Step 9: Image Compression.

It is mainly performed to save disc space by representing an image with a minimum number of bits. There are two kinds of image compression, and they are Lossy compression, Lossless compression.

In Lossy compression, some of the information is lost, and in Lossless compression, the image that will be re-constructed will be the same as the original version of the image. In the Lossless compression method, two techniques are being used: run-length coding and Huffman coding.

- $\quad$ Step 10: Color Image Processing.

Within 100 shades of grey, the human eye can only distinguish hundreds of thousands of different colors. The image contains more information. Using this information, one can simply analyze images such as object identification, extraction.

In the image processing approach, the image can be easily analyzed in the post-processing part. Images can be stored in computer memory, and the initial cost can vary.

The architecture of the Proposed System

In this study, Fig. 1 presents the proposed user-friendly plant disease detection system architecture, which has focused on three phases.

- $\quad$ Phase One: The data collected with the unique interaction of electronic devices.

- Phase Two: Analyze and process the data given by individual users for future disease prediction.

- Phase Three: Detect plant disease along with providing necessary information

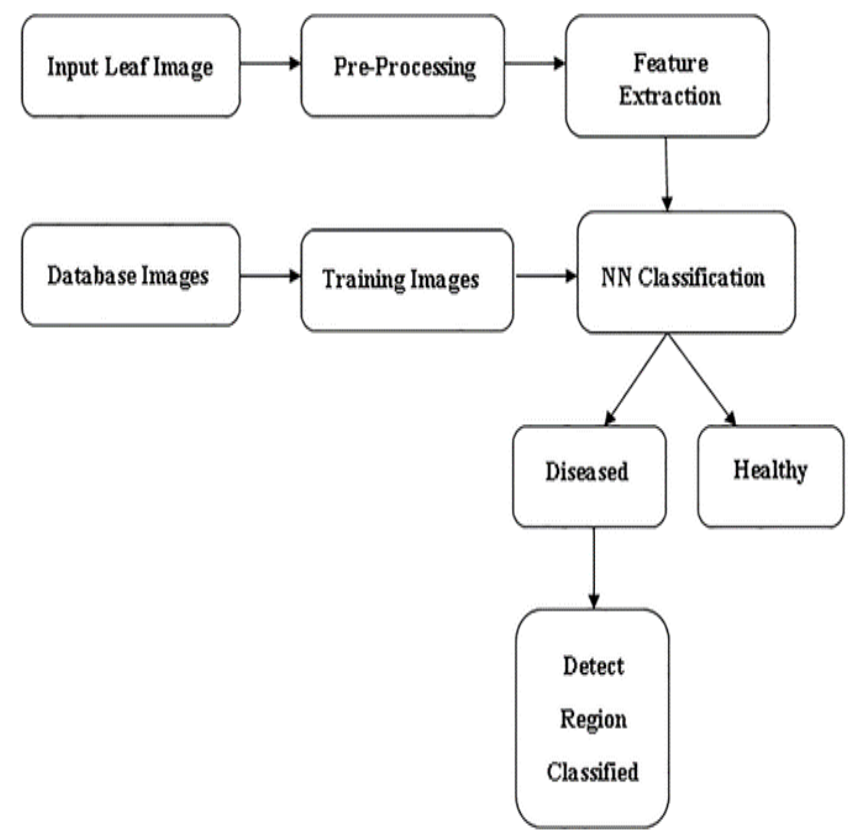

Fig. 1 Three phases of user-friendly plant disease detection system

Here, in Fig. 1,

Input Leaf Image $->$ Pre-Processing- > Feature Extraction -> NN Classification -> Diseased / Healthy. 


\section{Diseased -> Detect Region Classified.}

Database Images -> Training Images -> NN Classification.

The system will collect data through a user interface. Users can use electronic devices to input data. The data can be an image of the plant, such as the leaf of the plant. After collecting the image, it will be compiled with the trained model the authors already have. I $t$ will classify whether the plant is diseased or not. If it is healthy, it will be informed to the users. However, if it is not, then treatment plans and suggestions will be shown to the users. In this research, the researchers have used only two types of plants: Tomato and beans. In the case of Tomato, it will only show the two statuses (healthy, diseased). But, in the case of beans, it will show two types of state (healthy, diseased); in diseased, there are also three classifications (low affected, medium affected, and profoundly affected). The authors already have the data on the temperature of each area from previous years. So, based on those data, necessary actions will be suggested to the users. The data (image, output, and temperature-related data) will be stored in a cloud-based database such as Google Drive.

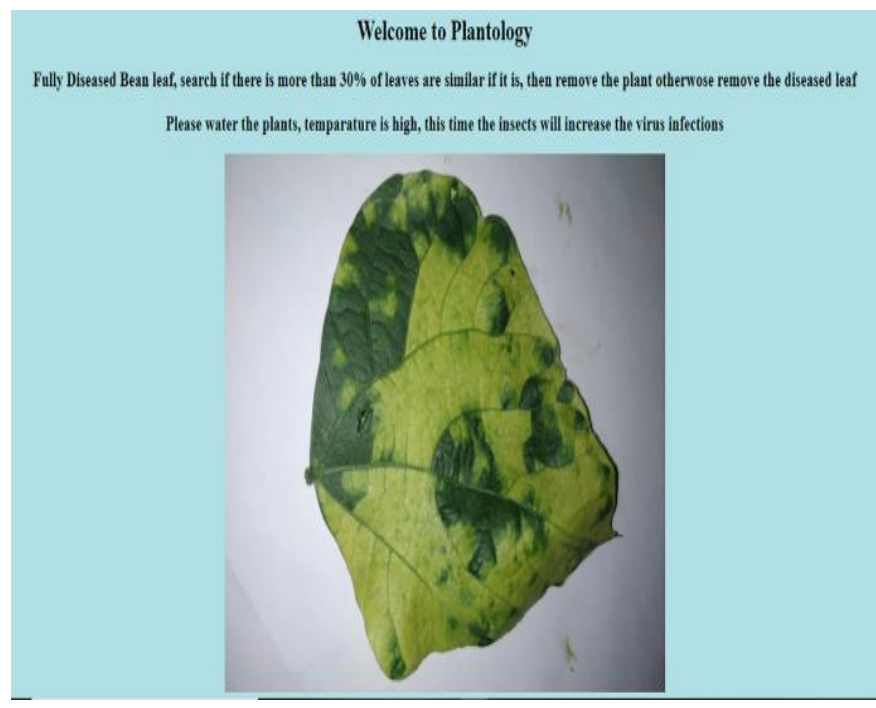

Fig. 2 Implementation of a user-friendly plant disease detection system.

Fig. 2 represents the implementation phase of the plant disease detection system.

At first, it welcomes the user to this 'Plantology' Application. It analyzes a given image and, after detection, provides output such as 'Fully Diseased Bean leaf, search if there is more than $30 \%$ of leaves are similar. If it is, then remove the plant; otherwise, remove the diseased leaf. Please water the plants, and the temperature is high; this time, the insects will increase the virus infections'.

\subsection{Brief system architecture}

\subsubsection{Convolutional Neural Network (CNN)}

This algorithm addresses classification problems and helps to recognize images. It is also used in identifying videos, recommendation systems, etc. There are four layers in this network.

Layer One: Convolution.

The features metric has been generated randomly in the convolutional layer and multiplied using the matric with the input image pixel-by-pixel values. Then, all the values are added and divided with the total number of feature pixels. Here, the feature pixel is generated randomly, and the weight is passed down for forwarding propagation. After that, the weight will be updated after Backpropagation. In this way, after completing the calculation, the final metric is found.

Layer Two: ReLu.

Rectifier Linear Unit is also known as ReLu, which works as one of CNN's activation functions. ReLu mainly helps remove the negative values from the result gained from Convolution Layer, which helps get only the primary feature value from the image. The ReLu equation can be explained when the input is less than zero; the output is equal to zero. When the input is greater than or equal to zero, the output is similar to the input.

Layer Three: Pooling.

There are many kinds of pooling functions, such as max pooling, average pooling, mean pooling, etc. For this algorithm, max pooling is widely used, and it has mainly $2 \times 2$ or $3 \times 3$ window size, where it only gives the maximum values from the metrics to generate only the most essential values from the data. It helps to shrink the input metrics. After passing the pooling layer, the final parameter is found, and that will be sent to the next layer.

Layer Four: Fully Connectedness (also known as Fully Connected Layer).

It converts the input metrics into a 1D array or a single list.

$\mathrm{CNN}$ can be sequential or functional. On CNN, some hidden layers are called convolutional layers, and they make a CNN. There can also be non-convolutional layers, but the base of a $\mathrm{CNN}$ is mainly the convolutional layers. This layer receives input, transforms it somehow, and shows the output after that. This output will be the input for the next layer. With this convolution layer, this transformation that passes through is called a convolution operation. Convolution layers can easily detect images and patterns, such as edges and images also. While using the convolutional layer, one needs to specify the number of filters that the layers will work with. These filters mainly detect the patterns. ReLu or Rectified Linear Activation Function transforms the summation of the weighted input from a particular node to the activation part and shows the output. To make training more comfortable and achieving better performance, it is highly needed. The pooling layer simplifies the data by reducing dimensionality. This also reduces training time and overfitting problems. In the fully connected layers, every neuron is connected to the next. In this stage, it mainly looks for those features, which accurately describe the particular classes. The result shows a probability with a single vector that is embodied in depth.

This algorithm is being used in different areas such as vehicle recognition systems, natural language processing tasks, computer vision, drug discovery, game playing, etc.

\subsubsection{Multi-Layer Convolutional Neural Network (MCNN)}

MCNN works just like CNN, it is just different from most of the other CNN architectures, and it consists of multilayers of the convolutional layer. It is also one of the deep learning models that are used for solving complex problems.

In the model described in the paper [8], they have been inspired by AlexNet architecture, which has six convolutional layers with six ReLU(Rectifier Linear Unit), three max-pooling layers, one flatten layer, and two dense or fully-connected layers. The first two convolutional layers' size is $(128,3 \times 3)$, the next 
two convolutional layers' size is $(256,3 \times 3)$, and last two convolutional layers' sizes are $(384,3 \times 3)$ and $(256,3 \times 3)$. In this model, the authors have used each of the max-pooling layers with a pool size of $(2 \times 2)$ and a dropout rate of 0.2 . The flatten layer helps the result set to convert into output form, and the dense or fully-connected layer supports it to turn into a 1D array or single list and uses SoftMax as the activation function.

\subsubsection{Faster Region-based Convolutional Neural Network (FRCNN)}

FRCNN is implemented to detect the object. It uses a search selective algorithm to discover the regions of interest and passes those to a ConvNet. Then it searches the areas that may be an object by uniting similar types of pixels and textures into multiple rectangular boxes. The search selective algorithm is calculated based on the feature map's output, which is found in the previous step.

After that, the ROI (region of interest) pooling layer is implemented to ensure that the standard and pre-defined output sizes are all accurate. Then, valid outputs are passed down to fully connected layers to be considered as inputs. In the final step, two output vectors are implemented to portend the observed object with a SoftMax classifier and modify bounding box localizations along with a linear regressor. Before training, the images have been annotated using the label box. After training, it produces a significant result with annotation.

In FRCNN, RPN (region proposal network) is used to reduce computation costs and detect images faster than before. RPN uses a feature map as input, which is generated by the network. It slides a $3 X 3$ sliding window over that map with the help of $\mathrm{K}$ anchor boxes. Besides, it performs this operation for each window. One gets an anchor box every time after each of the training phase finishes. Anchor box helps to improve the output of the detection and reduces cost. As for the training phase, one may consider the sliding window with a different scale and aspect ratio. In this case, anchor boxes help a lot, as it is scale and translation invariant.

After that, one will calculate loss using the loss function. There are also two kinds of loss functions here. One is Classification Loss, and the other is Regression Loss. To train the RPN, randomly 256 anchors will be selected and considered as a mini-batch. Then, the loss function will be computed using this.

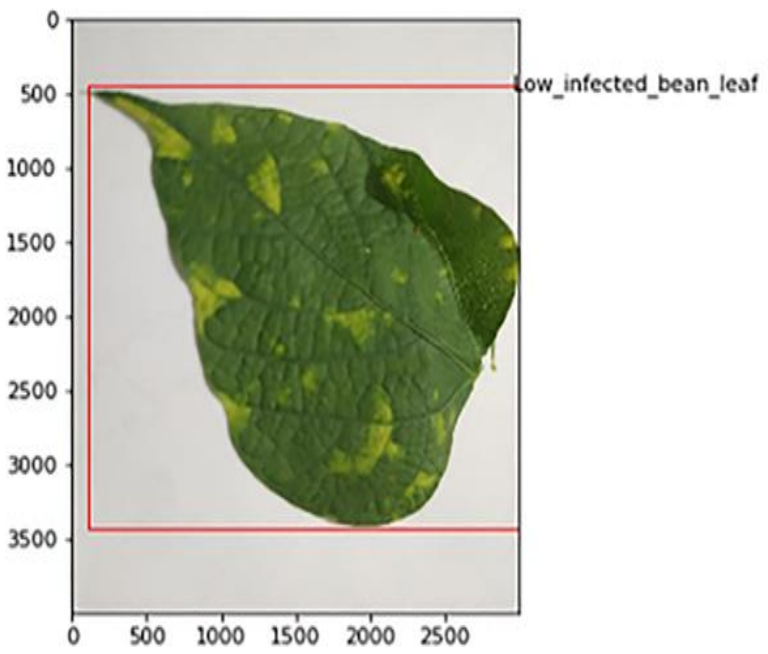

Fig. 3 The labeled image of the low infected leaf of bean
Fig. 3 indicates the labeled image of the low infected leaf of the bean

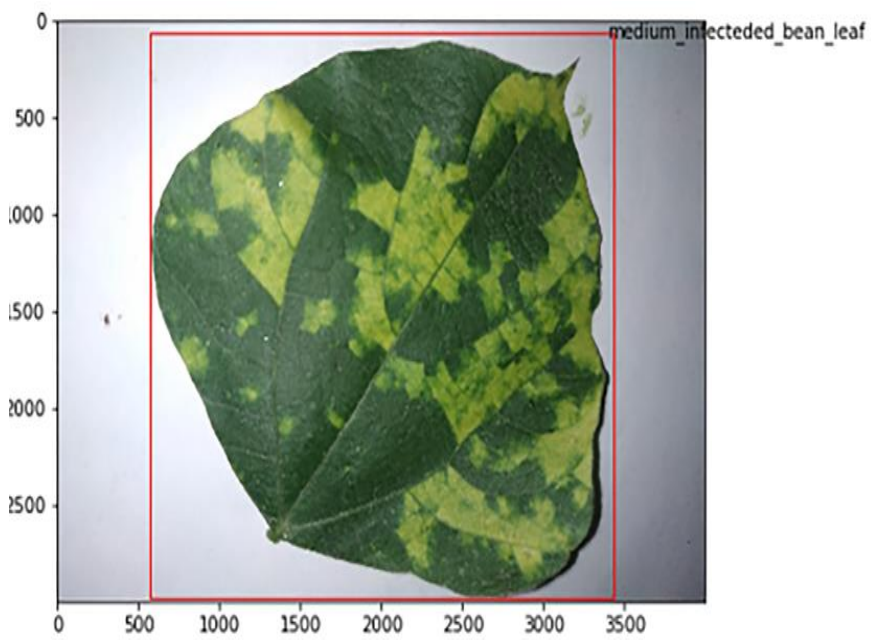

Fig. 4 The labeled image of the medium infected leaf of bean

Fig. 4 indicates the labeled image of the medium infected leaf of the bean.

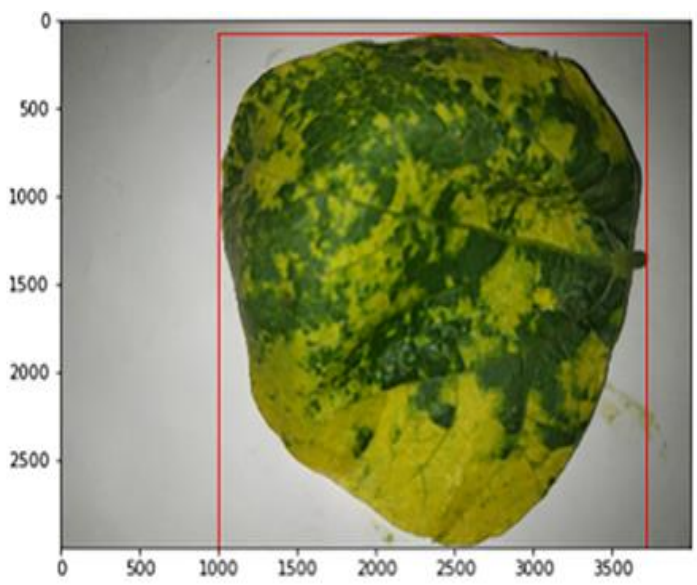

Fig. 5 The labeled image of the highly infected leaf of bean

Fig. 5 represents the labeled image of the highly infected leaf of bean

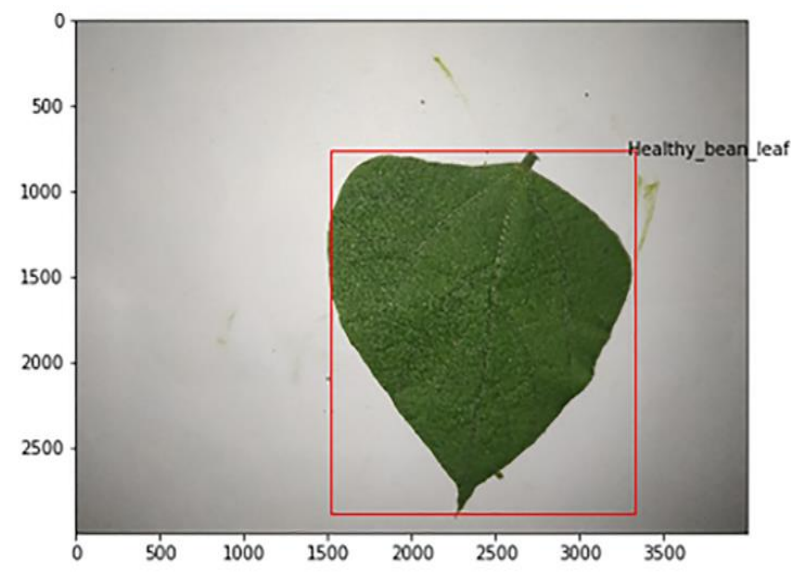

Fig. 6 The labeled image of the fully infected leaf of bean

Fig. 6 depicts the labeled image of a fully infected leaf of the bean. 


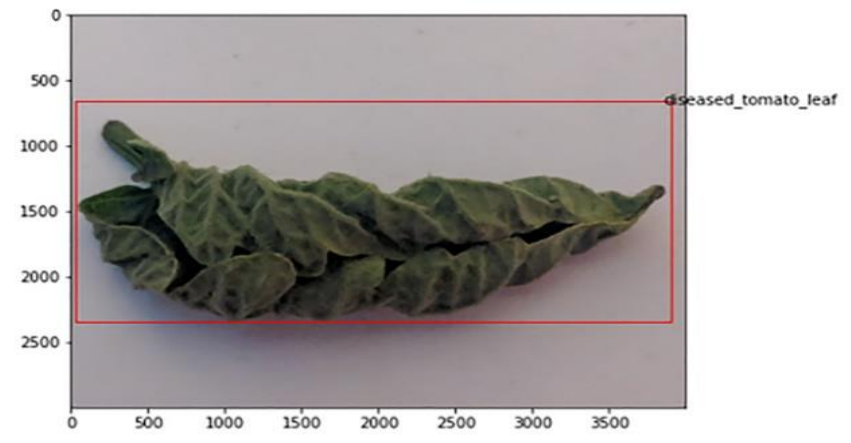

Fig. 7 The labeled image of the healthy leaf of bean

Fig. 7 represents the labeled image of the healthy leaf of bean

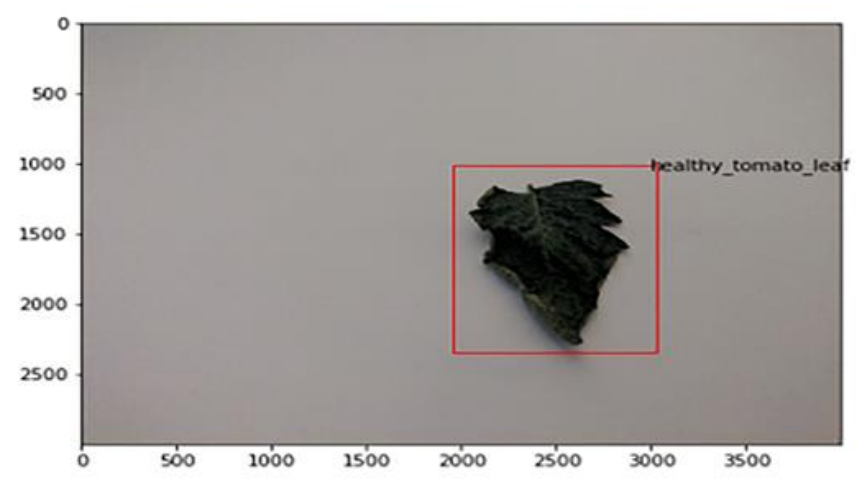

Fig. 8 The labeled image of the infected leaf of Tomato

Fig. 8 shows the labeled image of the infected leaf of Tomato

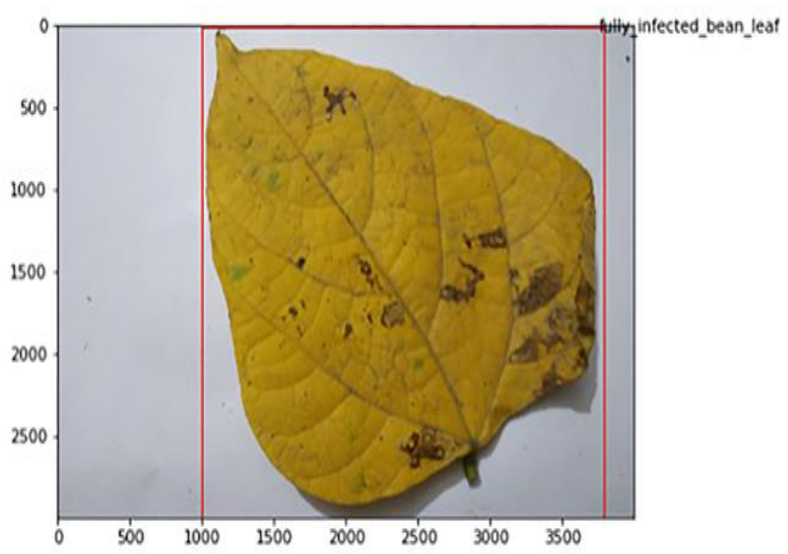

Fig. 9 The labeled image of the healthy leaf of the Tomato

Fig. 9 indicates the labeled image of a healthy leaf of Tomato.

\subsubsection{Modified Convolutional Neural Network (ModCNN)}

This model is a sequential model that consists of a few layers. The architecture of this model is shown in Fig. 10. In this model, at first, there are two convolution layers with the size of $(32,3 \mathrm{X} 3)$. Then comes the max-pooling layer with size $(2 \mathrm{X} 2)$. Again, comes two convolution layers with the size of $(64,3 \times 3)$. Then, a max-pooing layer with size $(2 \times 2)$. Now comes one convolution layer with the size of $(128,3 \mathrm{X} 3)$. Again, comes a max-pooling layer with size (2X2). At this stage, there will come a flattened layer. In the final stage, there will be two fully connected layers with a SoftMax activation function. This model will be implemented to process the images and detecting them accurately.

There are various optimizers, but the authors have used Adam optimizer, which manipulates the learning rate by changing momentum. For loss function, binary cross-entropy has been used, which generates multiple classes' loss function. The learning rate is 0.05 , and after the learning rate, the batch size was 32. In the study, the researchers have used 25 epochs. Though different numbers of epochs have been used through the research, at last, 25 epochs have succeeded in achieving the highest accuracy in both the training \& testing phase, which are respectively $98.52 \%$ and $97.69 \%$.

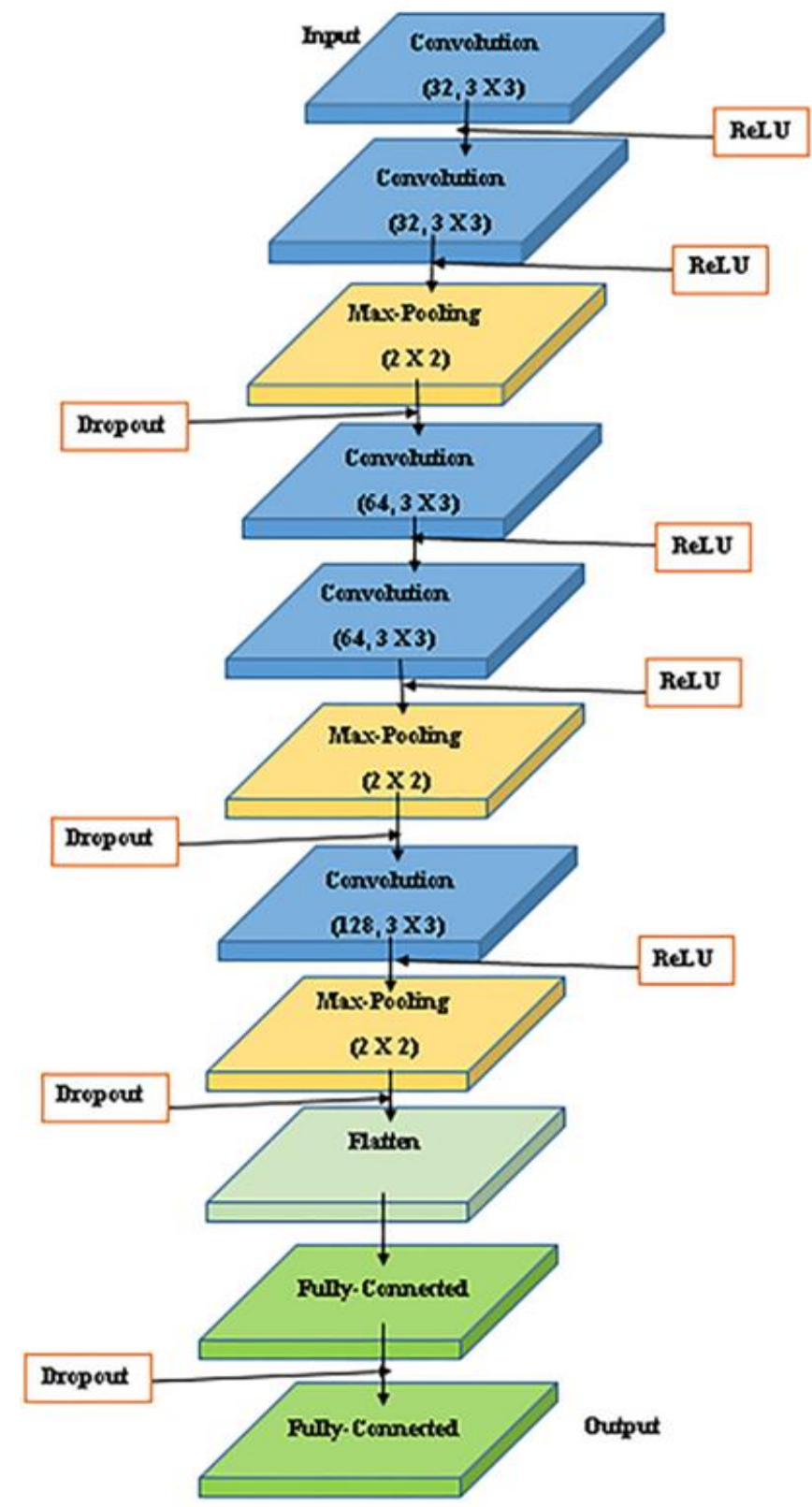

Fig. 10 The architecture model of the proposed ModCNN algorithm

\subsection{Environmental setup}

The authors have conducted this research using Anaconda Navigator (Jupyter Notebook) version 3.2 and have used python as a programming language. The laptop used to conduct this research and compile codes has 8 GB Ram, 4 GB Graphics, 256 
GB SSD, and the Intel is CORE i5. In the backend, h5py, Keras 2.0.3, NumPy, OpenCV-python, and sklearn have been used.

\subsection{Dataset and disease description}

In this research, two plant leaves have been used, and they are bean and Tomato. The images of leaves have been collected from Sher-E-Bangla Agricultural University under the supervision of Dr. Md. Belal Hossain, Professor, Department of Plant Pathology (Faculty of Agriculture). While collecting the images, white background has been used. There are a total of 1043 images of bean and tomato leaves. In the case of beans, there are 596 images where 213 images are healthy leaves, and 383 images are diseased leaves (71 images of low infected, 76 images of medium infected, 187 images of highly infected, and 49 images of fully infected). In the case of Tomato, there are a total of 447 images where there are 302 healthy images of leaves and 145 diseased images. The Tomato has 'Leaf Curl disease,' and the bean has 'Mosaic Virus.' But, while evaluating, the tomato leaf will only show whether it's diseased or healthy, but the bean leaf will show four stages when it's diseased (low, medium, high, and fully infected).

In this study, the researchers have worked with two diseases; one is tomato curl leaf, and the other is the bean mosaic virus disease. In tomato leaf curl disease, the leaf becomes curl due to the leaf curl virus's effect. In this case, the plant grows severely unbalanced, the size of the leaf decreases, excision in flowers, and so on. An insect named Aphid works as a carrier of this virus. On the other side, for bean leaf, when the mosaic virus occurs, the leaf shows some light green spots. The more virus affects the leaves, and the spots become bigger. In this case, the whitefly insect is the carrier of the mosaic virus.

At a temperature of 25 -35 degrees, Celsius is suitable for both carriers to outspread the Tomato and Bean leaves' virus. If the insect is reduced from the plant, the outspread of the virus will also decrease. For that reason, at that temperature, the plants must be watered, and pesticides must be used. The virus-affected leaf should be removed from the plant to protect the other leaves from the virus. If the temperature is $<25 \%$, the leaf is affected by the virus, and then the plant can be saved by removing the affected leaves. However, if it goes more than $40 \%$, the plant has to be cut down to protect other plants near it from the virus. In this study, the authors have built a modified CNN model that gives $97.69 \%$ testing accuracy in predicting Tomato and bean leaf diseases. The researchers have also collected temperaturerelated data of the Dhaka division from the previous years, which also shows the average temperature of each month. The model will take input of an image of either Tomato or bean leaf that will predict if it is diseased or not. If the leaf is diseased, then the model will take the month from the system automatically. It will check the temperature-related data of that month for that year, and if the temperature is higher than 35-degree coleus at that time, the model will suggest watering the plants and using insecticide. If the situation becomes more dangerous or gets out of hand, the user will be advised to visit any specialist for further treatments.

\section{Results and Discussions}

\subsection{Comparison of Performances}

There will be a comparison of detailed analysis among this study with some of the other authors' studies related to plant disease detection using various deep learning models.

\section{Test Accuracy of MCNN (\%)}

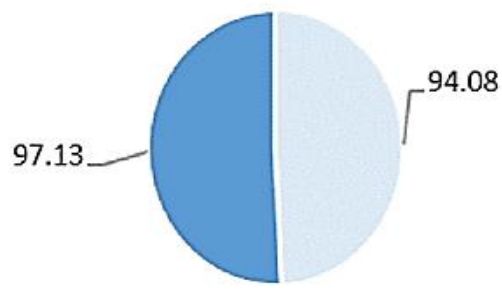

- Our Dataset $\quad$ " paper [9]

Fig. 11 Test accuracy of MCNN (\%) between this study \& paper [9]|

Comparison based on test accuracy in the MCNN algorithm between this study and paper [9]| has been represented in Fig. 11. This study has achieved $94.08 \%$ test accuracy white paper [9]| has scored $97.13 \%$. In this case, paper [9] has provided better performance than this study.

Comparison based on test accuracy in the FRCNN algorithm between this study and paper [10] has been indicated in Fig. 12. This study has achieved $60 \%$ test accuracy while paper [10] has scored $98.26 \%$. In this case, paper [10] has provided better performance than this study.

\section{Test Accuracy of Faster $\mathrm{RCNN}(\%)$}

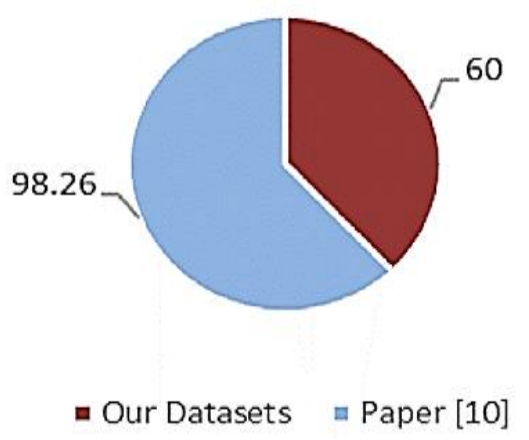

Fig. 12 Test accuracy of FRCNN (\%) between this study \& paper [10]

Comparison based on test accuracy in the case of CNN, MCNN, FRCNN, and Modified CNN algorithm among this paper and the other papers from the related work has been represented in Fig. 13. This paper has achieved $97.69 \%$ test accuracy using the proposed modified CNN, while paper [3] has scored $99.75 \%$. In this case, both of the papers have shown promising performances as both of them have used different types of algorithms and have still managed to score more than $95 \%$. 


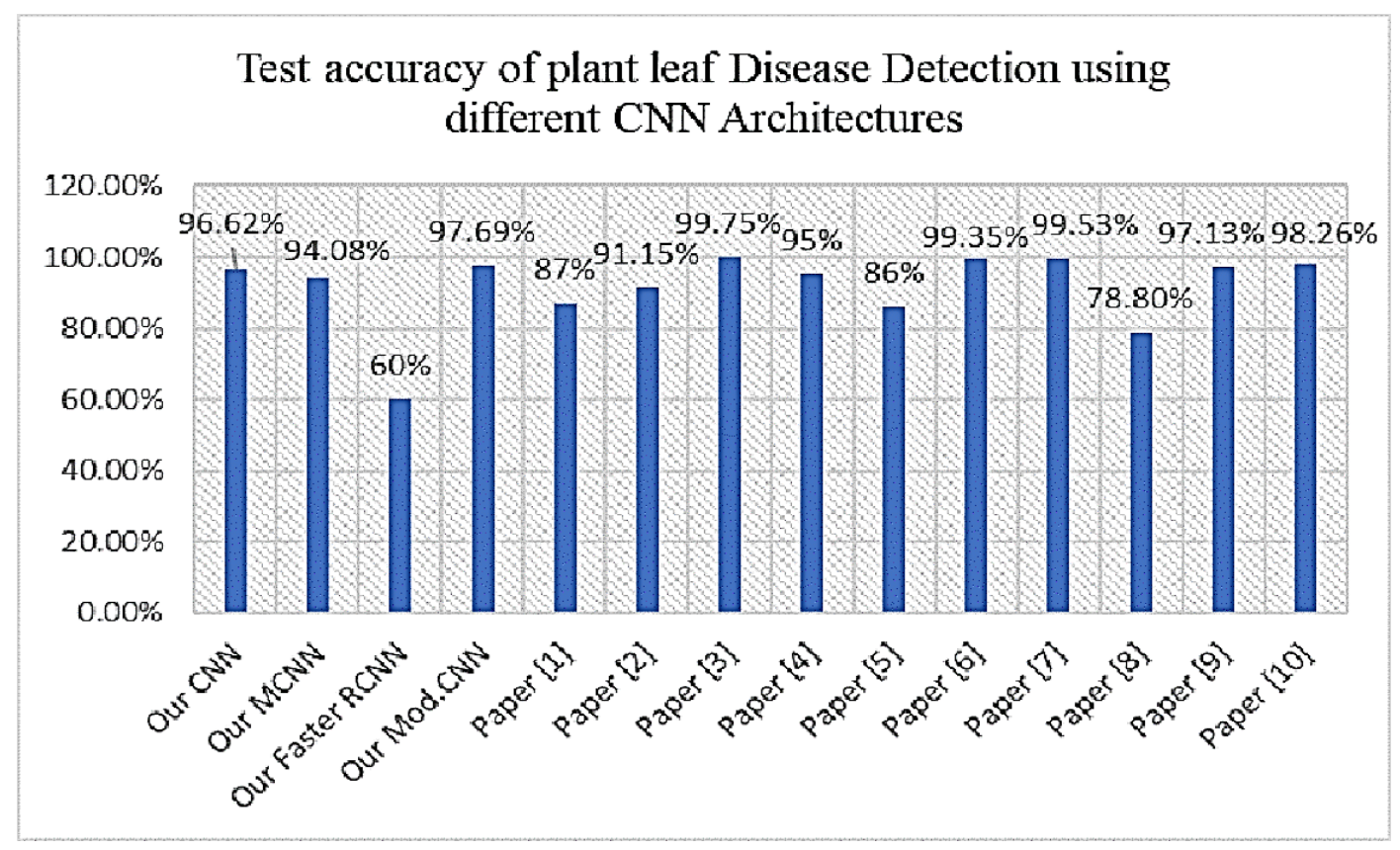

Fig. 13 Test accuracy of plant leaf disease detection using different $\mathrm{CNN}$ architectures

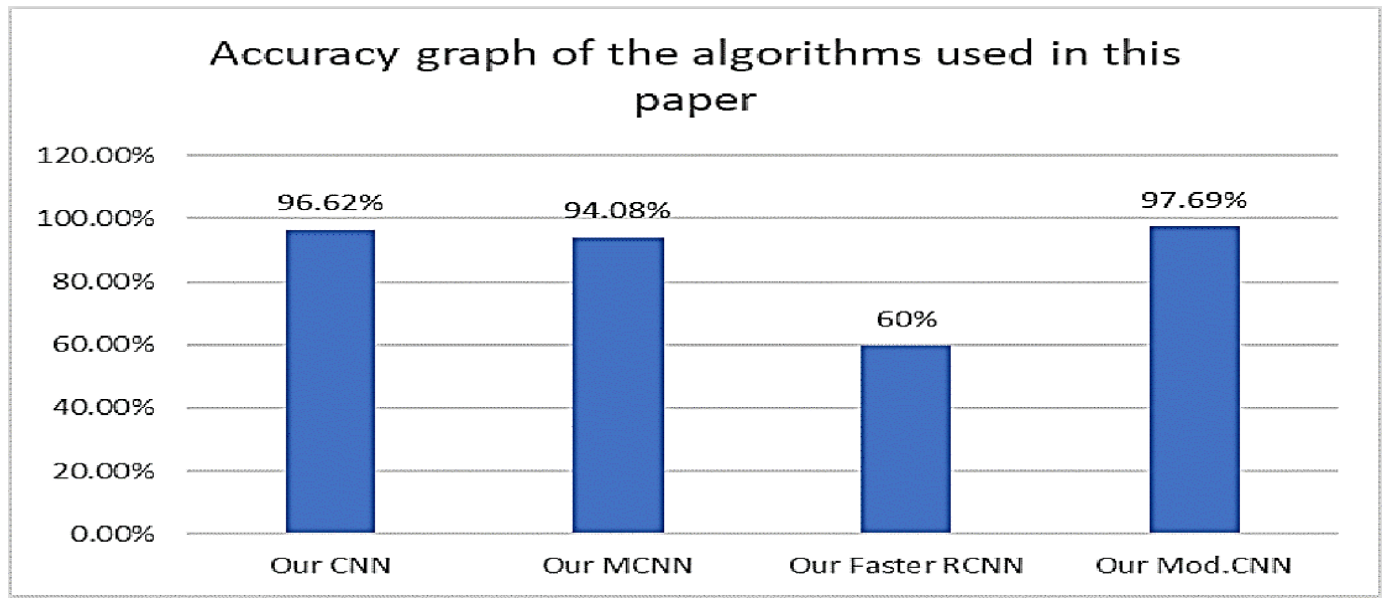

Fig. 14 Accuracy graph of the algorithms used in this study

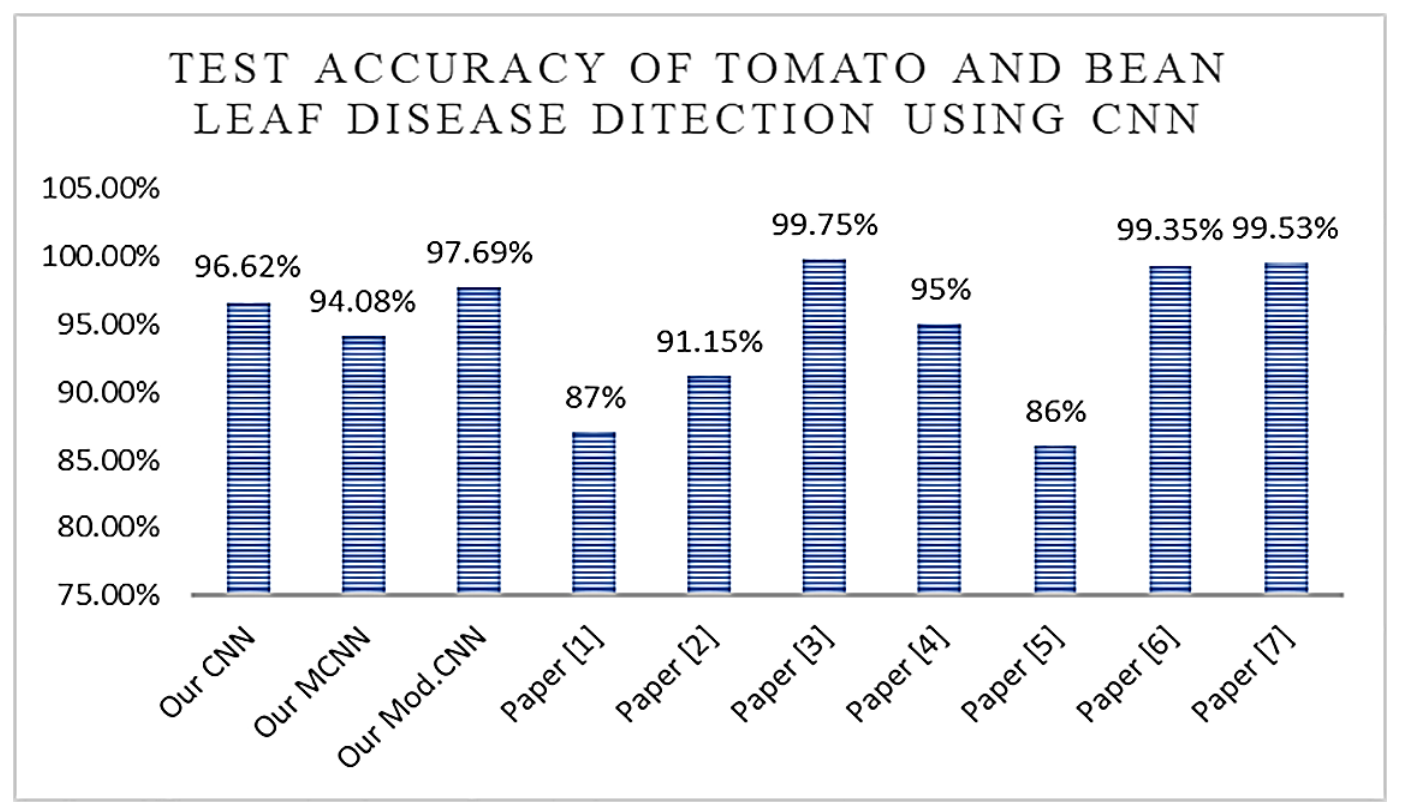

Fig. 15 Test accuracy of tomato and bean leaf disease detection using $\mathrm{CNN}$ 
F. Nihar et al. /JEA Vol. 02(01) 2021, pp 48-57

Table 1 Comparison table of used methods along with the test accuracy of related works

\begin{tabular}{|c|c|c|c|}
\hline References & Used methods & Datasets & Test Accuracy (\%) \\
\hline$[1]$ & CNN & Corn Leaf & 87 \\
\hline$[2]$ & TCCNN & Tomato leaf & 91.15 \\
\hline$[3]$ & CNN & 14 different plant leaves from Plant village & 99.75 \\
\hline$[4]$ & CNN & Plant village datasets & 95 \\
\hline$[5]$ & CNN with LVQ & Tomato leaf & 86 \\
\hline$[6]$ & CNN & 14 different corps from plant village & 99.35 \\
\hline$[7]$ & CNN & 25 different plants & 99.53 \\
\hline$[8]$ & CNN & Apple leaf & 78.8 \\
\hline$[9]$ & MCNN & Mango leaf & 97.13 \\
\hline$[10]$ & Faster RCNN & Rice leaf & 98.26 \\
\hline
\end{tabular}

Comparison based on the test accuracy of tomato and bean leaf disease detection using different types of CNN among this paper and the other papers from the related work has been shown in Fig. 15. This paper has achieved $97.69 \%$ test accuracy using the proposed modified CNN, while paper [3] has scored $99.75 \%$. In this case, both of the papers have shown outstanding performances as both of them have used different types of CNN and have still managed to score more than $95 \%$.

The comparison based on some other papers from the related works has been indicated in

Table 1. The factors which are considered here are used methods, datasets, and test accuracy in percentage. From

Table 1, it is clear that paper [3] has scored the highest test accuracy. It has used 14 different plant leaves from the plant village and achieved $99.75 \%$ accuracy by implementing CNN.

Table 2 indicates that the proposed ModCNN model provides the highest accuracy in both training \& testing phases among the models used for the dataset.

Table 2 Comparison table of accuracy based on the used methods to detect tomato \& bean leaf diseases

\begin{tabular}{|c|c|c|}
\hline \multicolumn{3}{|c|}{ Accuracy } \\
\hline & Train Accuracy & Test Accuracy \\
\hline CNN & $98.21 \%$ & $96.62 \%$ \\
\hline MCNN & $98.51 \%$ & $94.08 \%$ \\
\hline ModCNN & $98.52 \%$ & $97.69 \%$ \\
\hline Faster RCNN & $66 \%$ & $60 \%$ \\
\hline
\end{tabular}

\section{Conclusion and Future Work}

\subsection{Limitations}

Now, at this point, different types of questions can arise, such as why the accuracy is lower. Well, due to some limitations, the authors have encountered this type of situation.

While collecting data, the authors have faced image preprocessing steps due to image capture conditions as all of the images have been collected directly from the field, then sent to the pre-processing stage. In the pre-processing stage, some of the images were almost similar. Therefore, it was complicated to differentiate them to assign them to a particular class label. But it will not be an issue for the farmers who will use this model. The researchers have faced these kinds of problems for training the model, but in the farmer's case, they don't have to give an accurate image of their plant as the researchers did. They can provide raw data from the field, and the model which have been implemented and trained by the researchers will predict the disease accurately. The farmers don't have to train the model and go through the testing phase like the researchers, so at the user end, they will not face these kinds of problems in the future.

From the result of the algorithms used in our study, Mod$\mathrm{CNN}$ has provided the highest accuracy result though there is a limitation of collecting lots of data. For the other algorithms, such as FRCNN, which has produced the lowest test accuracy result. Nevertheless, it has been found that if there is a low amount of data, then FRCNN is not preferable for creating those models. If some developers or researchers want to expand this study, it will be better to get more data to increase the accuracy result for FRCNN. But for Mod-CNN, they can use our model and work with other crops and vegetables.

However, after facing all these issues, the authors have tried hard and achieved $97.69 \%$ accuracy and intend to increase accuracy in the future.

\subsection{Future Work}

This study's future work is to develop a user interface on android using a cloud-based storage system and apply other algorithms to improve the performances. This will add a new dimension and open another way in the agricultural arena to improve the system by including more interactive features. Twenty-four hours of the monitoring system for the plants through live video recording and live monitoring systems can be developed to notify the user via the internet system if there are any slight chances of infection in the leaf. In this way, the user can take any required action instantly.

\subsection{Conclusions}

The proposed system for detecting plant disease will improve the agricultural system's development, which will accurately predict whether a plant is infected or not. If not, it will be notified, but if affected, then the treatment plan will also be provided to help the user save the plant as soon as possible. This system allows the user to diagnose diseases earlier to take reasonable preventive measures beforehand and save plants. This is a time-effective approach as the users can easily do it within a short amount of time without visiting anywhere. It is costeffective, as it does not require any money to diagnose the disease. This system shows a better and promising future in the agricultural arena, and people need this system to save the plants from leading a happy, healthy, and comfortable life. 


\section{References}

[1] Barbedo, J.G., 2018. Factors influencing the use of deep learning for plant disease recognition. Biosystems engineering, 172, pp.84-91.

[2] Zhang, S., Huang, W. and Zhang, C., 2019. Three-channel convolutional neural networks for vegetable leaf disease recognition. Cognitive Systems Research, 53, pp.31-41.

[3] Too, E.C., Yujian, L., Njuki, S. and Yingchun, L., 2019. A comparative study of fine-tuning deep learning models for plant disease identification. Computers and Electronics in Agriculture, 161, pp.272-279.

[4] Singh, K.K., 2018, November. An artificial intelligence and cloud based collaborative platform for plant disease identification, tracking and forecasting for farmers. In 2018 IEEE International Conference on Cloud Computing in Emerging Markets (CCEM) (pp. 49-56). IEEE.

[5] Sardogan, M., Tuncer, A. and Ozen, Y., 2018, September. Plant leaf disease detection and classification based on CNN with LVQ algorithm. In 2018 3rd International Conference on Computer Science and Engineering (UBMK) (pp. 382-385). IEEE.

[6] Mohanty, S.P., Hughes, D.P. and Salathé, M., 2016. Using deep learning for image-based plant disease detection. Frontiers in plant science, 7, p.1419.

[7] Dalakouras, A., Wassenegger, M., Dadami, E., Ganopoulos, I., Pappas, M.L. and Papadopoulou, K., 2020. Genetically modified organism-free RNA interference: exogenous application of RNA molecules in plants. Plant physiology, 182(1), pp.38-50.

[8] Jiang, P., Chen, Y., Liu, B., He, D. and Liang, C., 2019. Real-time detection of apple leaf diseases using deep learning approach based on improved convolutional neural networks. IEEE Access, 7, pp.59069-59080.

[9] Singh, U.P., Chouhan, S.S., Jain, S. and Jain, S., 2019. Multilayer convolution neural network for the classification of mango leaves infected by anthracnose disease. IEEE Access, 7, pp.43721-43729.

[10] Zhou, G., Zhang, W., Chen, A., He, M. and Ma, X., 2019. Rapid detection of rice disease based on FCM-KM and faster R-CNN fusion. IEEE Access, 7, pp.143190143206.

[11] Bhuiyan, M., Rahman, A., Ullah, M. and Das, A.K., 2019. iHealthcare: Predictive model analysis concerning big data applications for interactive healthcare systems. Applied Sciences, 9(16), p.3365.

[12] Cynthia, S.T., Hossain, K.M.S., Hasan, M.N., Asaduzzaman, M. and Das, A.K., 2019, December. Automated detection of plant diseases using image processing and faster R-CNN algorithm. In 2019 International Conference on Sustainable Technologies for Industry 4.0 (STI) (pp. 1-5). IEEE.

[13] Paul, S., Joy, J.I., Sarker, S., Ahmed, S. and Das, A.K., 2019, December. An Unorthodox Way of Farming Without Intermediaries Through Blockchain. In 2019 International Conference on Sustainable Technologies for Industry 4.0 (STI) (pp. 1-6). IEEE.

[14] Islam, S., Khan, S.I.A., Abedin, M.M., Habibullah, K.M. and Das, A.K., 2019, July. Bird species classification from an image using vgg-16 network. In Proceedings of the 2019 7th International Conference on Computer and Communications Management (pp. 38-42).

[15] Kaur, S., Pandey, S. and Goel, S., 2018. Semi-automatic leaf disease detection and classification system for soybean culture. IET Image Processing, 12(6), pp.10381048. 